\title{
Changes in SLIT2 expression are associated with the migration of human ovarian clear cell carcinoma cells
}

\author{
CUEI-JYUAN LIN ${ }^{1 *}$, WAY-REN HUANG ${ }^{2 *}$, CHIA-ZHEN WU ${ }^{3}$ and RUO-CHIA TSENG ${ }^{3}$ \\ ${ }^{1}$ Department of Laboratory Medicine, Keelung Chang Gung Memorial Hospital, Keelung 20401; \\ ${ }^{2}$ GLORIA Operation Center, National Tsing Hua University, Hsinchu 30013; \\ ${ }^{3}$ Department of Molecular Biology and Human Genetics, Tzu Chi University, Hualien 97004, Taiwan, R.O.C.
}

Received January 8, 2021; Accepted May 5, 2021

DOI: $10.3892 / 01.2021 .12812$

\begin{abstract}
Ovarian clear cell carcinoma (OCCC) is characterized by a poor survival of patients, which is mainly due to metastasis and treatment failure. Slit guidance ligand 2 (SLIT2), a secreted protein, has been reported to modulate the migration of neural cells and human cancer cells. However, the effect of changes in SLIT2 expression on the regulation of cell migration in OCCC remains unknown. The present study examined alterations in SLIT2 expression using OCCC cell models, including low- and high-mobility SKOV3 cells, as well as OCCC tissues. DNA methylation analysis suggested that promoter hypermethylation was responsible for the low expression levels of SLIT2 in OCCC cells. The demethylating agent 5-Aza-deoxycytosine was able to restore SLIT2 expression at both the mRNA and protein levels in high-mobility SKOV3 cells that harbored the relevant methylated promoter. Overexpression of SLIT2 inhibited the migration of high-mobility OCCC cells, as well as decreased the protein expression levels of $\beta$-catenin, phosphorylated (p)AKT and snail family transcriptional repressor 1 (SNAI1). On the other hand, knockdown of SLIT2 increased the migration of low-mobility OCCC cells, and enhanced the protein expression levels of $\beta$-catenin, pAKT and SNAI1. Overall, the results of the present study provided evidence that low expression levels of SLIT2 were associated with increased OCCC cell migration, and that SLIT2 may act as a suppressor gene of cancer cell migration.
\end{abstract}

\section{Introduction}

Epithelial ovarian cancer (EOC) is consistently one of the leading causes of cancer-associated mortality with a mortality

Correspondence to: Dr Ruo-Chia Tseng, Department of Molecular Biology and Human Genetics, Tzu Chi University, 701 Zhongyang Road, Hualien 97004, Taiwan, R.O.C.

E-mail: rctseng8@mail.tcu.edu.tw

*Contributed equally

Key words: ovarian clear cell carcinoma, tumor suppressor, methylation, slit guidance ligand 2, migration rate of $\sim 9 \%$ in Taiwan and various other areas of the world $(1,2)$. EOC has several subtypes with different origins, multiple molecular characteristics and a range of outcomes (3). EOC consists of five histological subtypes, namely serous, mucinous, clear cell, endometrioid and transitional cell/Brenner tumor subtypes (4). Ovarian clear cell carcinoma (OCCC) is a distinct type of ovarian cancer, and is associated with both a poor survival and resistance to platinum-based chemotherapy (3). OCCC is the second most common EOC subtype in Taiwan and Japan (2), whereas it ranks fourth in North America (5). Despite significant efforts to develop new targeted diagnostic and therapeutic approaches aimed at decreasing mortality, these have been largely unsuccessful, as metastasis remains the main cause of mortality and accounts for $\sim 90 \%$ of all OCCC-associated deaths $(6,7)$.

Metastasis is a multistep process that is a significant cause of cancer-associated mortality in humans (8). Therefore, identifying genes and molecular pathways in OCCC that are associated with metastasis may lead to advances in therapeutics. Our previous studies have revealed that low expression levels of slit guidance ligand 2 (SLIT2) are associated with a poor survival and promote esophageal cancer metastasis $(9,10)$. SLIT2 is a secreted glycoprotein of the SLIT family and is the human orthologue of the Drosophila Slit2 protein (11). SLIT2 is the ligand of the receptor roundabout guidance receptor 1 (ROBO1), which is known to participate in intercellular signal transduction via GTPase activation protein (12). Moreover, this signaling serves an important role in cell migration $(9,12)$. In addition, SLIT/ROBO signaling has been revealed to be involved in the development of a number of organs, including the heart and organs of the reproductive tract and nervous system (13).

Our previous study has indicated that SLIT2 may be a candidate tumor suppressor that may be silenced in epithelial tumors of the aerodigestive tract via genetic deletion and epigenetic promoter hypermethylation (10). Furthermore, the epigenetic silencing of SLIT2 has been observed in serous ovarian cancer (14-16). A previous study has indicated low SLIT2 expression in EOC samples compared with in the normal human ovarian surface epithelium (17). Additionally, SLIT2 expression can significantly decrease the invasion and migration of endometrial carcinoma cells (18). Moreover, injecting exogenous $\mathrm{ROBO} 1$-expressing cells into nude mice 
decreases the size of breast tumors (19). However, to the best of our knowledge, there are no published studies that have investigated changes in SLIT2/ROBO1 signaling in OCCC. Therefore, the present study performed a range of molecular analyses on human normal and malignant OCCC samples, as well as on SKOV3 cells that are able to form OCCC. The current findings revealed that SLIT2 may be a potential molecular target for the treatment of human OCCC.

\section{Materials and methods}

Cell culture. The SKOV3 low-mobility (SKOV3-L) and high-mobility (SKOV3-H) cell lines were kindly provided by Dr Lu-Hai Wang (Institute of Molecular and Genomic Medicine, National Health Research Institute, Miaoli, Taiwan) (20). SKOV3 cells were maintained in RPMI-1640 medium with $10 \%$ FBS (both Thermo Fisher Scientific, Inc.). All cells were incubated at $37^{\circ} \mathrm{C}$ in a humidified atmosphere with $5 \% \mathrm{CO}_{2}$.

Western blot analysis. Cells were lysed in RIPA buffer $(0.05 \mathrm{M}$ Tris- $\mathrm{HCl}, \mathrm{pH} 7.4,0.15 \mathrm{M} \mathrm{NaCl}, 0.25 \%$ deoxycholic acid, $1 \%$ NP-40, 1 mM EDTA, $0.5 \mathrm{mM}$ DTT, $1 \mathrm{mM}$ phenylmethylsulfonyl fluoride, $5 \mu \mathrm{g} / \mathrm{ml}$ leupeptin and $10 \mu \mathrm{g} / \mathrm{ml}$ aprotinin) on ice, and then centrifuged at $15,000 \mathrm{x} \mathrm{g}$ at $4^{\circ} \mathrm{C}$ for $5 \mathrm{~min}$. The protein concentration was estimated by a BSA standard curve. Subsequently, SDS gel loading buffer (60 mM Tris base, $2 \%$ SDS, $10 \%$ glycerol and 5\% $\beta$-mercaptoethanol) was added to the samples, and $50 \mu \mathrm{g}$ protein/lane was separated by $8 \%$ SDS-PAGE. The proteins were electro-blotted onto Immobilon-P membranes (EMD Millipore) using transfer buffer. The membranes were blocked with 5\% skim milk in phosphate-buffered saline with $0.1 \%$ Tween-20 (PBST) for $1 \mathrm{~h}$ at room temperature. Immunoblotting was performed using primary anantibodies against SLIT2 (cat. no. AB5701; 1:800; MilliporeSigma), $\beta$-catenin (cat. no. 9582; 1:1,000; Cell Signaling Technology, Inc.), phosphorylated (p)AKT (cat. no. sc-7985-R; 1:500; Santa Cruz Biotechnology, Inc.), AKT (cat. no. 4691; 1:800; Cell Signaling Technology, Inc.) and snail family transcriptional repressor 1 (SNAI1; cat. no. 3895; 1:500; Cell Signaling Technology, Inc.). $\beta$-actin (cat. no. GTX109639; 1:1,000; GeneTex, Inc.) was used as an internal control to confirm that equal amounts of proteins had been loaded onto the gel. The membranes were subsequently probed with a horseradish peroxidase-conjugated secondary antibody (cat. no. \#12-371, 1:5,000; MilliporeSigma) for $1 \mathrm{~h}$ at room temperature. The bands were visualized using a western blot chemiluminescence reagent (MilliporeSigma).

Reverse transcription-quantiative PCR (RT-qPCR). Total RNA was prepared from tumor cells using TRIzol ${ }^{\circledR}$ reagent (Invitrogen; Thermo Fisher Scientific, Inc.). cDNA was synthesized using Oligo(dT)18 primer (Genedirex, Inc.), dNTPs (Protech, Inc.), RT buffer (Bioline, Inc.) and SuperScript ${ }^{\mathrm{TM}}$ Reverse Transcriptase (Invitrogen; Thermo Fisher Scientific, Inc.) according to the manufacturer's instructions. The mRNA expression levels of SLIT2 were measured using the Applied Biosystems StepOne ${ }^{\mathrm{TM}}$ Real-Time PCR System (Thermo Fisher Scientific, Inc.) and $\mathrm{SYBR}^{\circledR}$-Green (Thermo Fisher Scientific, Inc.) according to the manufacturer's instructions with $\beta$-actin as an internal control. The mRNA expression levels were evaluated using the following primers: SLIT2 forward, 5'-GGTGTCCTCTGTGATGAAGAG-3' and reverse, 5'-GTGTTTAGGACACACACCTCG-3'; and $\beta$-actin forward, 5'-GGCGGCACCACCATGTACCCT-3' and reverse 5'-AGG GGCCGGACTCGTCATACT-3'. Reactions were performed in a volume of $25 \mu \mathrm{l}$ with $1 \mu \mathrm{l}$ cDNA and 0.25 pmol primers. The PCR protocol involved 35 cycles of three sequential steps: $95^{\circ} \mathrm{C}$ for $30 \mathrm{sec}, 58^{\circ} \mathrm{C}$ for $30 \mathrm{sec}$ and $72^{\circ} \mathrm{C}$ for $30 \mathrm{sec}$. The mRNA expression levels were calculated using the $2^{-\Delta \Delta \mathrm{Cq}}$ method $\left(\Delta \mathrm{Cq}=\mathrm{Cq}_{\mathrm{SLIT} 2}-\mathrm{Cq}_{-\beta \text {-actin }}\right)(21)$.

TissueScan array. The present study was performed after approval from the research ethics committee review board of the Tzu-Chi Hospital (approval no: 101-04; Hualien, Taiwan). TissueScan Ovarian Cancer Panels (cat. nos. HORT502 and HORT504; OriGene Technologies, Inc.) were purchased on 4 April, 2018, to examine the mRNA expression levels of SLIT2 in cDNA samples that had been prepared from normal ovarian and ovarian tumor tissue samples. Briefly, the TissueScan plate was removed from $-20^{\circ} \mathrm{C}$ storage and allowed to warm at room temperature. A PCR pre-mix was prepared containing $\mathrm{SYBR}^{\circledR}$ Green master mix, the aforementioned primers and double-distilled $\mathrm{H}_{2} \mathrm{O}$. A total of $25 \mu \mathrm{l}$ of the PCR pre-mix was added to each well in the 96-well TissueScan plate. The PCR was performed as aforementioned.

Methylation-specific PCR (MSP) assay. The methylation status of the promoter region of the SLIT2 gene was assessed by treating cells with sodium bisulfite, followed by an MSP assay. A total of $500 \mathrm{ng}$ genomic DNA was denatured at $95^{\circ} \mathrm{C}$ for 5 min and incubated with $\mathrm{NaOH}$ (final concentration, $0.2 \mathrm{M}$ ) at $37^{\circ} \mathrm{C}$ for $15 \mathrm{~min}$. Hydroquinone $(10 \mathrm{nM}$; Sigma-Aldrich; Merck KGaA) and sodium bisulfite (3 M; Sigma-Aldrich; Merck KGaA) were added and incubated at $50^{\circ} \mathrm{C}$ for $18 \mathrm{~h}$. Subsequently, modified DNA was purified using a Microcon YM-50 DNA purification column (EMD Millipore). Treatment of genomic DNA with sodium bisulfite converts unmethylated but not methylated cytosine residues to uracil residues, which are then converted to thymidine residues during the subsequent PCR step. The primers and PCR conditions were as described previously (9). A hypermethylated gene was defined as a gene in tumor cells from which the methylated PCR products were amplified more than the unmethylated PCR products (10).

5-aza-2'-Deoxycytidine (5-aza-dC) treatment. In total, $1 \times 10^{5}$ cancer cells were seeded in a $100-\mathrm{mm}$ culture dish before 5-aza-dC treatment. Cancer cells were allowed to double three times (mean duration, $144 \mathrm{~h}$ ) in the presence of $5 \mu \mathrm{mol} / 1$ 5-aza-dC (Sigma-Aldrich; Merck KGaA) at $37^{\circ} \mathrm{C}$. Subsequently, the cells were collected and subjected to MSP, RT-qPCR and western blot analysis, as aforementioned.

Analysis of SLIT2 knockdown, overexpression and treatment with SLIT2 protein. Short hairpin RNA (shRNA) plasmids, consisting of shRNA-control (non-targeting) and shRNA-SLIT2, were purchased from the RNAi Core Lab of Academia Sinica. The empty pCMV6 and pCMV6-SLIT2 overexpression vectors were obtained from OriGene Technologies, Inc. The SLIT2 knockdown was performed 
in SKOV3-L cells, whereas the SLIT2 overexpression was performed in SKOV3-H cells. A total of $1 \times 10^{5}$ cancer cells were transfected with $4 \mu \mathrm{g}$ shRNA-control, shRNA-SLIT2, pCMV6 or pCMV6-SLIT2 using Lipofectamine ${ }^{\circledR} 2000$ transfection reagent (Thermo Fisher Scientific, Inc.) for $4 \mathrm{~h}$ according to the manufacturer's instructions. Following a 48-h incubation, the cells were subjected to RT-qPCR and western blot analysis as aforementioned.

Human SLIT2 protein was purchased from Abcam. A total of $1 \times 10^{5}$ cells were treated with $5 \mathrm{ng} / \mathrm{ml} \mathrm{SLIT2}$ protein at $37^{\circ} \mathrm{C}$ for $48 \mathrm{~h}$, and the results were analyzed by migration assay.

Gap closure assay. The gap closure assay was conducted using a culture insert (Ibidi GmbH), and SKOV3-L and SKOV3-H cells were used in this assay. The cells used for the gap closure assays were transfected with the empty vector, SLIT2 overexpression plasmid, shRNA-control or shRNA-SLIT2, as aforementioned. The cells were serum-starved for $16 \mathrm{~h}$ before conducting the migration experiment. A cell-free gap of $500-\mu \mathrm{m}$ was created by removing the culture insert, and serum-free medium was added after the wounds were created. The cell-free gaps that remained after $12 \mathrm{~h}$ of culture were photographed under a light microscope and measured using ImageJ software (Version 1.51, National Institutes of Health). The area of the open wound was calculated. A total of three independent experiments were performed for each cell type.

Transwell migration assay. Transwell assays were performed to determine the migratory ability of the various types of SKOV3 cells transfected with SLIT2 overexpression or knockdown plasmids, as aforementioned. The Transwell system (Falcon; Corning Life Sciences) consisted of upper and lower chambers. The cells seeded in the upper chamber were able to migrate through the membrane to the lower chamber. In total, $3 \times 10^{5}$ cells were seeded into the upper chamber in serum-free RPMI-1640 medium and the lower chamber was filled with RPMI-1640 medium with 10\% FBS. After incubation for $24 \mathrm{~h}$ at $37^{\circ} \mathrm{C}$, the cells that were attached to the reverse side of the Transwell membrane were stained using crystal violet for $30 \mathrm{~min}$, and total cells were counted under a light microscope at x100 magnification. In total, three independent experiments were performed for each cell type.

Adhesion assay. An appropriate number of 6-well microplates were pre-coated with extracellular matrix (ECM) proteins (Sigma-Aldrich; Merck $\mathrm{KGaA}$ ) for $16 \mathrm{~h}$ at $4^{\circ} \mathrm{C}$ and were then blocked with 1\% BSA (Sigma-Aldrich; Merck $\mathrm{KGaA}$ ) at $37^{\circ} \mathrm{C}$ for $1 \mathrm{~h}$. Subsequently, $6 \times 10^{4}$ cells/well were seeded in the pre-coated wells in RPMI-1640 medium containing $10 \% \mathrm{FBS}$ and were then incubated in a rotating incubator at $37^{\circ} \mathrm{C}$ for $2 \mathrm{~h}$ to recover. Next, the cells were incubated for $30 \mathrm{~min}$ at $37^{\circ} \mathrm{C}$ in $5 \% \mathrm{CO}_{2}$, followed by three washes with PBS to remove any unattached cells. Subsequently, the remaining attached cells were fixed and stained with $1 \%$ crystal violet/MeOH for $10 \mathrm{~min}$ at room temperature. Finally, the cells were lysed using DMSO and the absorbance was measured at $550 \mathrm{~nm}$. The absorbance at $550 \mathrm{~nm}$ was proportional to the number of cells attached to the pre-coated ECM wells. In total, three independent experiments were performed for each cell type.
Data collection. The gene expression profiling and methylation profiling datasets were selected from the National Center of Biotechnology Information Gene Expression Omnibus (GEO; http://www.ncbi.nlm.nih.gov/geo/) database based on the keywords 'ovarian cancer' and 'Homo sapiens', and with the following inclusion criteria: i) Ovarian clear cell carcinomas[title]; ii) ovarian cancer[title], clear; iii) the dataset contained >50 samples; and iv) the dataset contained survival data. Datasets using serum and cell line samples were excluded. A total of two gene expression profiling (GSE8841 and GSE65986) and one methylation profiling (GSE51820) datasets were selected (22-24). The 83 ovarian tumor samples of the GSE8841 dataset contained 16 clear cell samples. The GSE65986 dataset contained 55 epithelial ovarian cancer samples, including 25 clear cell samples. The GSE51820 dataset contained 96 ovarian cancer samples, including 13 clear cell and 4 normal ovarian surface epithelium samples. In GSE65986 and GSE8841, the low expression group was defined as the expression value of SLIT2 < mean-1SD, and the other samples were defined as the high expression group (25). In the GSE51820 dataset, hypermethylation of the SLIT2 gene was defined as a probes value $>$ mean +1 SD of the OCCC samples (26).

Statistical analysis. The results of the RT-qPCR, gap closure and Transwell assays were analyzed using an unpaired Student's t-test. The Kaplan-Meier method was used to construct progression-free survival curves, and then a comparison was performed using the log-rank test. $\mathrm{P}<0.05$ was considered to indicate a statistically significant difference. SPSS version 19.0 (IBM Corp.) was used for all statistical analyses.

\section{Results}

Aggressive phenotype of OCCC cells and promoter methylation of SLIT2. OCCC cell models, which consisted of SKOV3-L and SKOV3-H cells, were used in the various experiments. A number of different cancer cell properties, including the cell migration rate and cell adhesion, were compared between SKOV3-L and SKOV3-H cells (Fig. 1). The gap closure rates of SKOV3-L and SKOV3-H cells were measured in order to assess their migratory ability. The SKOV3-H cells had a significantly higher migratory ability compared with the SKOV3-L cells (Fig. 1A). The adhesion of the SKOV3-L and SKOV3-H cells was also evaluated. The results demonstrated that SKOV3-H cells had a significantly decreased adhesive capacity compared with SKOV3-L cells, as determined via a cell adhesion assay (Fig. 1B).

Whether the expression levels of SLIT2 were altered in OCCC cells was further examined by measuring the protein expression levels of SLIT2 in SKOV3-L and SKOV3-H cells. The results indicated that there was lower SLIT2 expression in the SKOV3-H cells compared with that in the SKOV3-L cells (Fig. 1C). Subsequently, whether the low SLIT2 protein expression was due to downregulated SLIT2 mRNA expression was investigated. Consistent with the SLIT2 protein results, significantly lower SLIT2 mRNA expression was detected in the SKOV3-H cells compared with in the SKOV3-L cells (Fig. 1D). To determine if there was an epigenetic effect associated with the expression of the SLIT2 gene, DNA methylation 


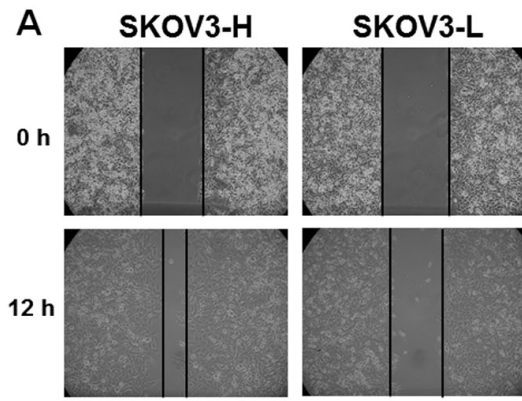

C

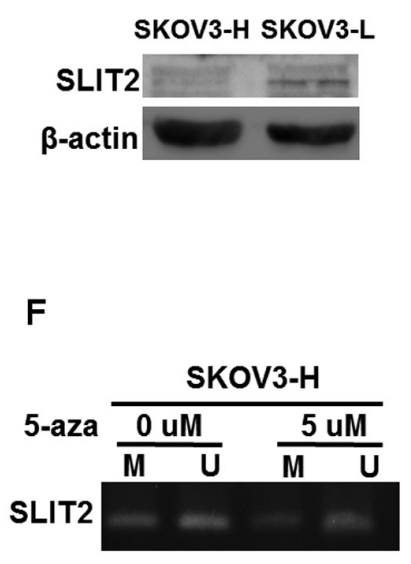

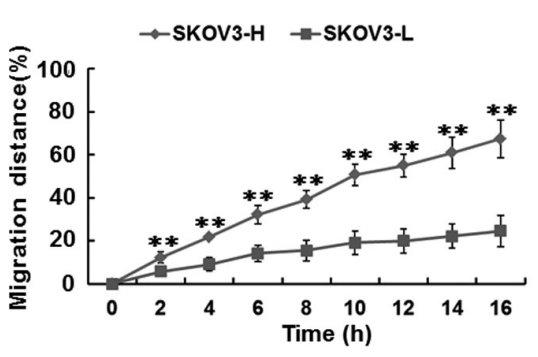

B
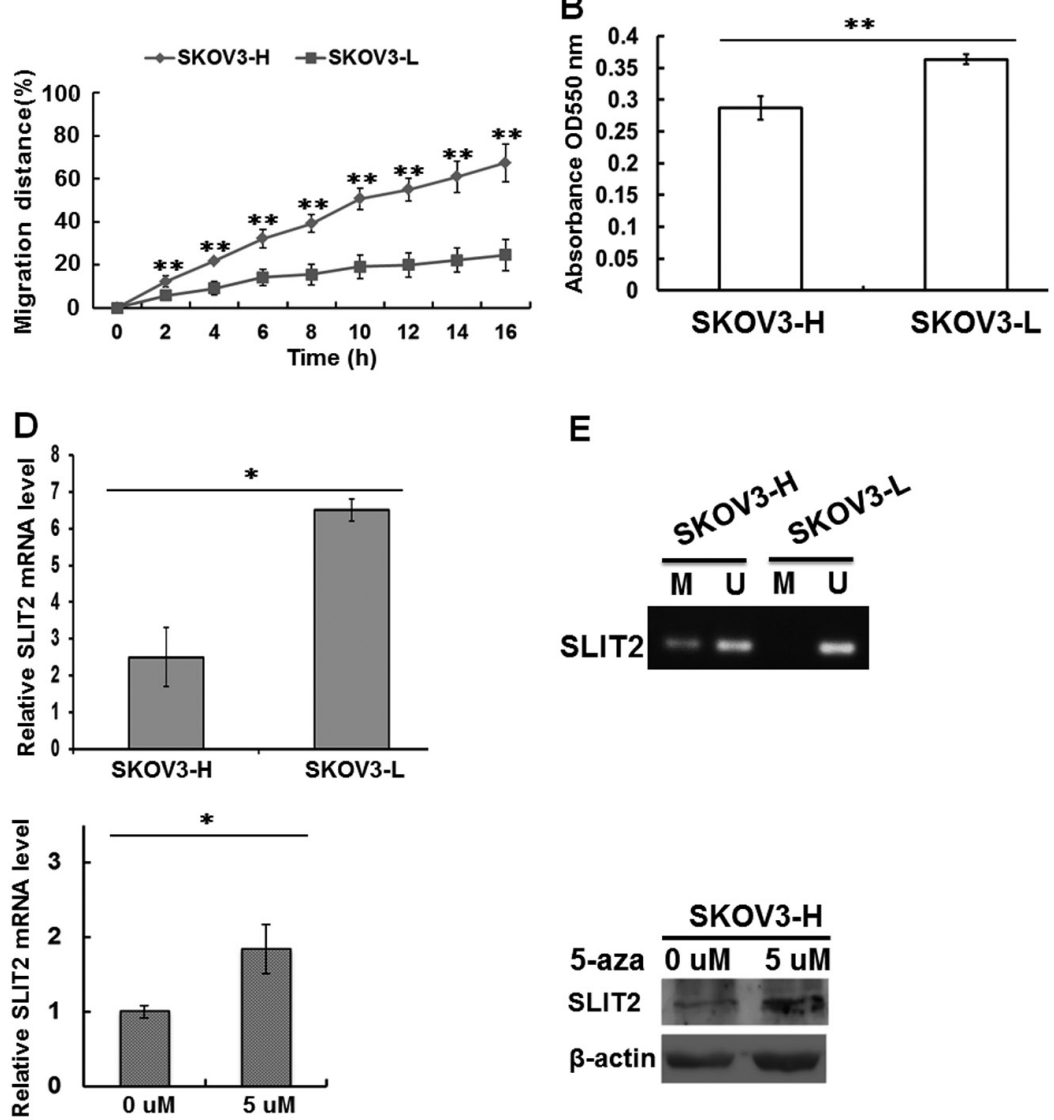

E
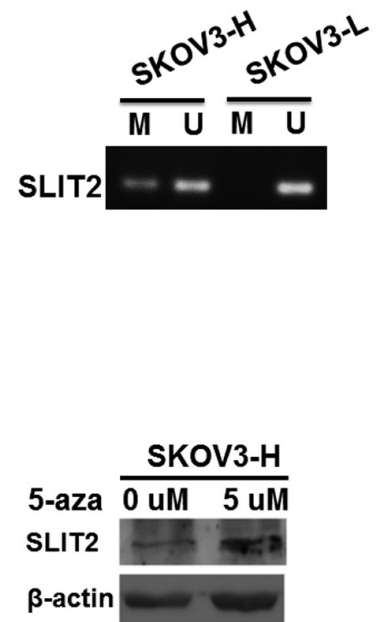

Figure 1. Mobility of ovarian clear cell carcinoma cells and epigenetic alteration of SLIT2. (A) Gap closure and (B) adhesion assays were conducted using SKOV3-L and SKOV3-H cells. (C) Protein expression levels of SLIT2. (D) mRNA expression levels of SLIT2. (E) DNA hypermethylation of the SLIT2 gene. (F) Effects of the treatment of SKOV3-H cells with the demethylating agent 5-aza-dC. The primer sets used for amplification are designated as ' $U$ ' for the unmethylated gene or ' $\mathrm{M}$ ' for the methylated gene. The P-value for each analysis was obtained using a two-tailed unpaired t-test. Quantitative data are presented as the mean \pm SD from three independent experiments. ${ }^{*} \mathrm{P}<0.05$ and ${ }^{* *} \mathrm{P}<0.01$ vs. SKOV-L. SLIT2, slit guidance ligand 2; SKOV3-L, low-mobility SKOV3 cells; SKOV3-H, high-mobility SKOV3 cells; 5-aza-dC, 5-aza-2'-deoxycytidine; OD, optical density.

assays of the SLIT2 gene were conducted on the two types of SKOV3 cells. DNA hypermethylation of the SLIT2 gene was observed in SKOV3-H cells, whereas a normal degree of methylation was observed in SKOV3-L cells (Fig. 1E).

It was then evaluated whether the low expression levels of SLIT2 were caused by the DNA methylation present in the SKOV3-H cells. The demethylating agent 5-aza-dC was used to treat SKOV3-H cells. As shown in Fig. 1F, demethylation of the SLIT2 gene by 5 -aza-dC treatment was able to increase SLIT2 mRNA and protein expression in SKOV3-H cells.

To confirm whether the hypermethylation of SLIT2 was associated with patients with OCCC, the publicly available GSE51820 dataset was analyzed. This methylation profile of 96 primary ovarian cancer samples included 13 clear cell ovarian cancer samples and 4 normal ovarian surface epithelium samples. A SLIT2 methylation probes value of OCCC higher than the mean of the 4 normal ovarian surface epithelium samples + 1SD indicated hypermethylation. Based on this criterion, the frequency of SLIT2 methylation was between 31 and $85 \%$ in patients with OCCC (Table SI).

Migration of OCCC cells is suppressed by overexpression of SLIT2 or by the addition of purified SLIT2 protein. Our previous studies have revealed that low SLIT2 expression is able to promote the migration of lung and esophageal cancer cells $(9,10)$. Therefore, it was hypothesized that SLIT2 may act as a cell migration suppressor in OCCC. To verify the role of SLIT2 reactivation in ovarian cancer cell migration, gap closure and Transwell assays were performed using SKOV3-H cells in the presence and absence of the ectopic expression of SLIT2. The overexpression of SLIT2 resulted in high SLIT2 protein expression in SKOV3-H cells (Fig. 2A). Moreover, a significant decrease in the migration of SKOV3-H cells was observed with SLIT2 overexpression compared with the control cells (Fig. 2B and C).

Since SLIT2 is a secreted glycoprotein, a gap closure assay was used to examine whether purified SLIT2 protein was able to affect the mobility of SKOV3-H cells. The results demonstrated that addition of purified SLIT2 protein $(5 \mathrm{ng} / \mathrm{ml})$ was able to significantly decrease the migratory capacity of SKOV3-H cells compared with the control cells (Fig. 2D).

Knockdown of SLIT2 increases the motility of OCCC cells. To further confirm the presence of a reciprocal association between SLIT2 expression and cell motility in OCCC, shRNA technology was used to knock down the SLIT2 gene in the SKOV3-L cells, which expressed SLIT2 at a high level and exhibited a low level of migration in vitro prior to transfection. Using western blot analysis, it was found that SLIT2-knockdown in SKOV3-L cells markedly inhibited the protein expression 
A

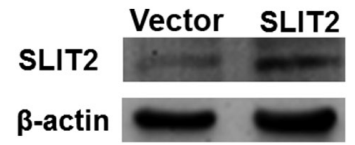

B
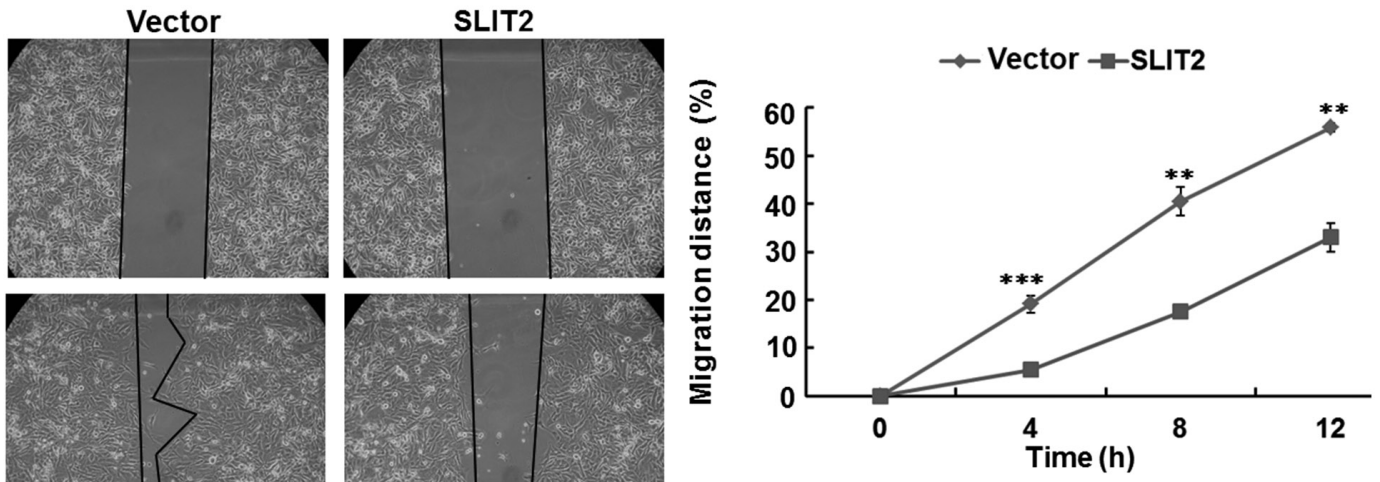

$12 \mathrm{~h}$
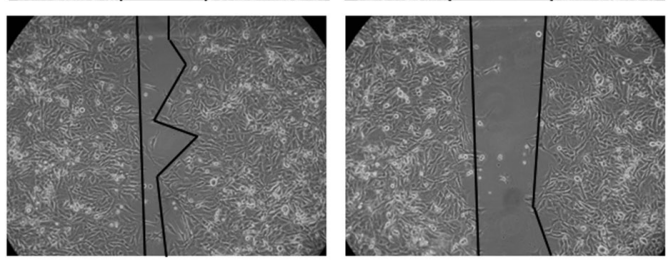

C
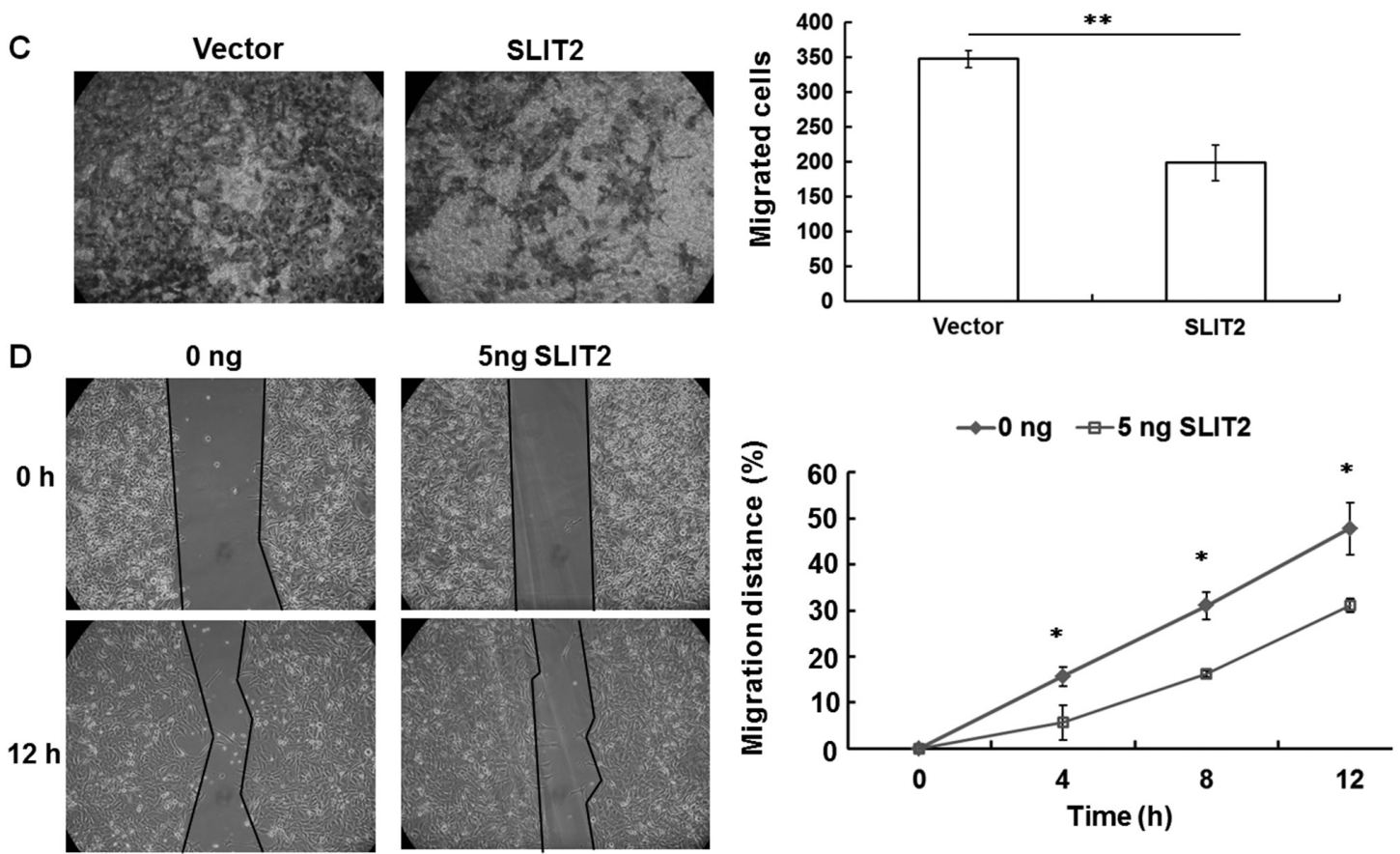

Figure 2. Overexpression of SLIT2 inhibits the migratory ability of SKOV3-H cells. (A) Protein expression levels of SLIT2 in SKOV3-H cells were measured via western blotting after overexpression of SLIT2. The migratory ability was assessed using (B) gap closure and (C) Transwell assays. (D) Cell migratory ability, as measured via a gap closure assay, was suppressed in SKOV3-H cells following the addition of purified SLIT2. The P-value for each analysis was obtained using a two-tailed unpaired t-test. Quantitative data are presented as the mean $\pm \mathrm{SD}$ from three independent experiments. Original magnification, $\mathrm{x} 100$. " $\mathrm{P}<0.05$, ${ }^{* *} \mathrm{P}<0.01$ and ${ }^{* * * *} \mathrm{P}<0.001$ vs. SLIT2. SLIT2, slit guidance ligand 2; SKOV3-L, low-mobility SKOV3 cells; SKOV3-H, high-mobility SKOV3 cells.

levels of SLIT2 compared with those in cells transfected with the shRNA-control (Fig. 3A). Next, the motility of these knockdown cells was determined using gap closure and Transwell assays. As shown in Fig. 3B and C, SKOV3-L cells transfected with shSLIT2-2 exhibited a significantly greater migratory capacity compared with control-treated SKOV3-L cells.

$\beta$-catenin/pAKT/SNAI1 involvement in the SLIT2-mediated mobility of OCCC cells. The epithelial-mesenchymal transition (EMT) of cancer cells is a fundamental feature of tumor invasion and metastasis (27). The transcription factors $\beta$-catenin and SNAI1, together with hyperactivation of the AKT signaling pathway, have been reported to be involved in the EMT process (28). To determine whether the
$\beta$-catenin/pAKT/AKT/SNAI1 axis was involved in the mechanism underlying SLIT2-mediated migration of OCCC cells, the protein expression levels of $\beta$-catenin, pAKT/AKT and SNAI1 were measured in the SLIT2-knockdown SKOV3-L cells and in SKOV3-H cells overexpressing SLIT2. SLIT2-knockdown SKOV3-L cells exhibited higher protein expression levels of $\beta$-catenin, pAKT and SNAI1 compared with the control cells (Fig. 4). Conversely, overexpression of SLIT2 in SKOV3-H cells resulted in decreased protein expression levels of $\beta$-catenin, pAKT and SNAI1 compared with the empty vector cells (Fig. 4).

Low SLIT2 expression occurs in human OCCC and is associated with a poor prognosis. The aforementioned results 
A Control shSLIT2-1 shSLIT2-2

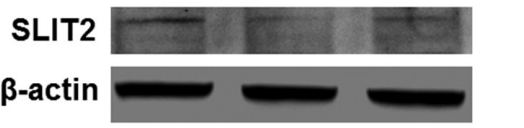

B

Control
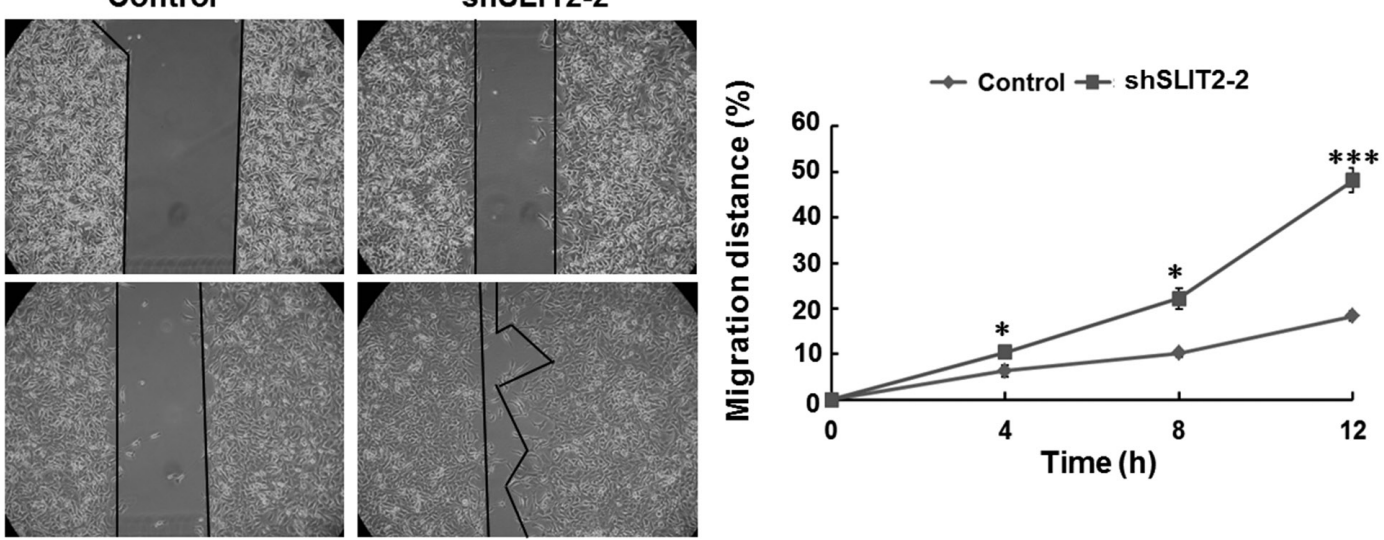

C
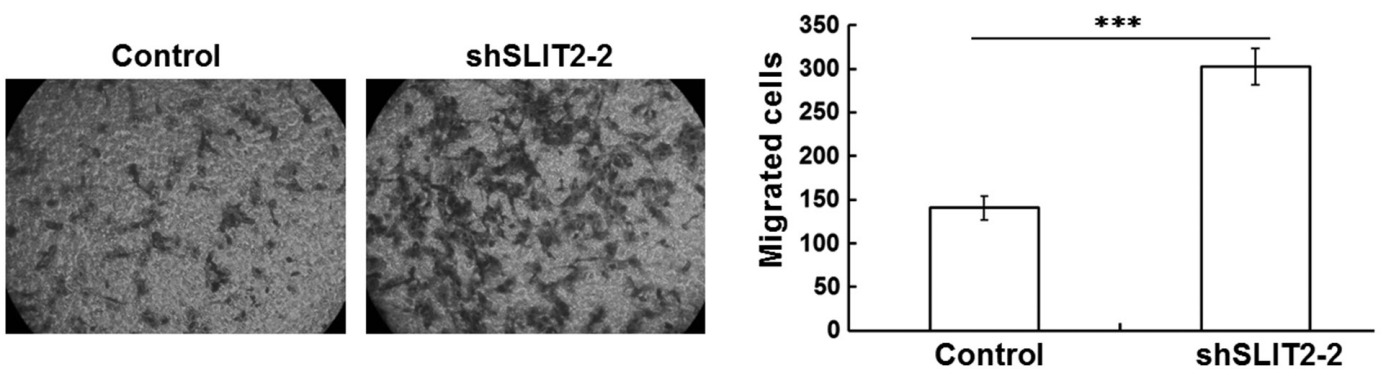

Figure 3. Knockdown of SLIT2 increases the migratory ability of SKOV3-L cells. (A) Protein expression levels of SLIT2 were measured via western blotting in SKOV3-L cells in the presence of shSLIT2. The migratory ability was assessed using (B) gap closure and (C) Transwell assays. The P-value for each analysis was obtained using a two-tailed unpaired t-test. Quantitative data are presented as the mean \pm SD from three independent experiments. Original magnification, $x 100 .{ }^{*} \mathrm{P}<0.05$ and ${ }^{* * * *} \mathrm{P}<0.001$ vs. control. SLIT2, slit guidance ligand 2; SKOV3-L, low-mobility SKOV3 cells; SKOV3-H, high-mobility SKOV3 cells; sh, short hairpin RNA.

indicated that SLIT2 expression was associated with the migration of OCCC cells in vitro. However, to the best of our knowledge, no studies have examined the mRNA expression levels of SLIT2 in OCCC tissue samples and their association with the clinical prognosis of patients. Therefore, RT-qPCR was performed to measure SLIT2 mRNA expression in two commercial array panels (HORT102 and HORT104), which contained 96 cDNA samples from 14 normal ovarian tissues and 82 ovarian tumor tissues including five OCCC samples (Table SII). The results demonstrated that four of the OCCC tissue samples had lower SLIT2 mRNA expression compared with the normal ovarian tissue samples (Fig. 5A).

To confirm whether low SLIT2 expression was associated with prognosis in patients with OCCC, survival curves were drawn using the Kaplan-Meier method. The SLIT2 mRNA expression of OCCC samples present in the publicly available GSE8841 and GSE65986 microarray datasets $(22,23)$ were evaluated. There were 16 OCCC samples collected by the GSE8841 project and 25 OCCC samples collected by the GSE65986 project (Table SIII). The results demonstrated that 17\% (7/41) of tumors exhibited low expression levels of SLIT2. It was found that the median survival time was 51 months in the low SLIT2 expression group and 89 months in the high SLIT2 expression group. Moreover, high SLIT2 expression was associated with a longer progression-free survival in patients with OCCC (Fig. 5B).

\section{Discussion}

To further understand the effects of changes in the expression levels of SLIT2 in OCCC, the present study performed a molecular analysis of OCCC cells, which included measuring the SLIT2 mRNA and protein expression levels and assessing the DNA methylation status of the SLIT2 gene. The current study identified an epigenetic mechanism that appeared to be associated with OCCC and involved SLIT2 gene silencing. Furthermore, the present study provided evidence that higher expression levels of SLIT2 may inhibit the progression of OCCC.

It is known that tumor suppressor genes can be inactivated by both genetic and epigenetic changes (29). In the present study, epigenetic silencing of SLIT2 by hypermethylation was observed, as determined by treating OCCC cells with 5-aza-dC. Consistent with these results, it was also revealed that the frequency of SLIT2 methylation ranged between $31-85 \%$ in 13 patients with OCCC from the GSE51820 project (Table SI). In addition, a study by Dong et al (16) reported that hypermethylation of the SLIT2 gene was frequently observed in patients with serous ovarian cancer. However, the possibility that somatic mutations of SLIT2 may also be implicated in carcinogenesis remains to be determined. Thus, detailed mutational analysis on the SLIT2 gene is required in order to obtain 


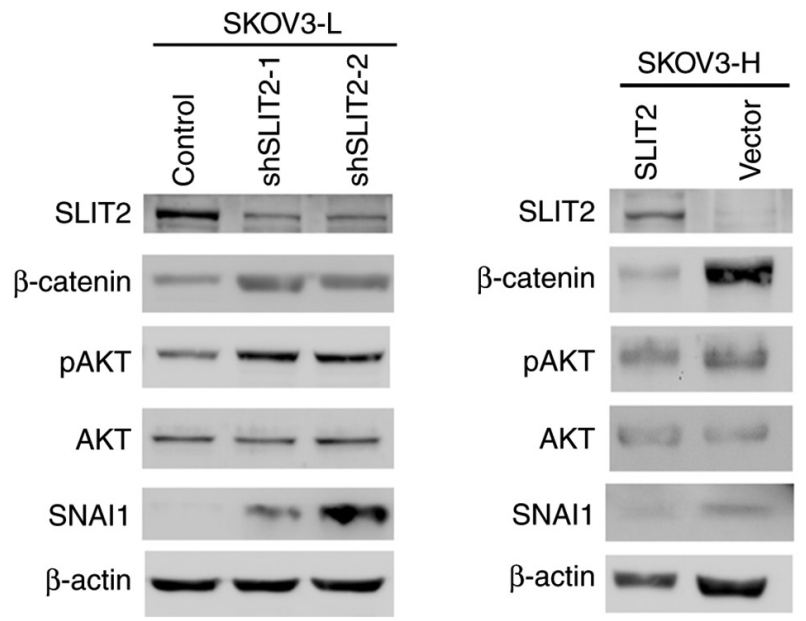

Figure 4. Protein expression levels of $\beta$-catenin, pAKT/AKT and SNAI1 were examined following SLIT-knockdown or overexpression in ovarian clear cell carcinoma cells. p, phosphorylated; SNAI1, snail family transcriptional repressor 1; SLIT2, slit guidance ligand 2; SKOV3-L, low-mobility SKOV3 cells; SKOV3-H, high-mobility SKOV3 cells; sh, short hairpin RNA.

an improved overall understanding of OCCC tumorigenesis. Qiu et al (14) found that decreased SLIT2 expression was significantly associated with SLIT2 promoter hypermethylation in 66 ovarian cancer samples. Conversely, Dai et al (15) found that SLIT2 was widely expressed in human normal and malignant ovarian tissue microarrays (4 adult granular cell tumor, 5 dysgerminoma, 8 adenocarcinoma, 5 teratoma malignant change, 6 yolk sac tumor, 20 mucinous adenocarcinoma and 136 serous adenocarcinoma), although the malignant ovarian tissue microarrays did not include OCCC samples. The present results provided evidence that lower SLIT2 expression was associated with epigenetic silencing of SLIT2. However, the lack of immunohistochemical analysis of SLIT2 in other subtypes, such as high-grade serous carcinoma or endometrial carcinoma, was a limitation of the present study. The role of SLIT2 in other histological subtypes, such as high-grade serous carcinoma, should be further investigated in future studies.

Previous studies have reported that SLIT2 may be a tumor suppressor gene $(10,14,16)$; however, to the best of our knowledge, the anti-migration properties of this gene have not yet been reported for human OCCC. The present findings demonstrated that the motility of OCCC cells was decreased when SLIT2 was overexpressed, while SLIT2-knockdown resulted in an increase in cell motility. Consistent with the current findings, Dickinson et al (30) revealed that inhibiting SLIT/ROBO activity induced an increase in the migration of primary luteal cell cultures. Moreover, another study by Dickinson et al (31) found that blocking SLIT/ROBO activity resulted in decreased apoptosis of SKOV3 cells. Jeon et al (32) observed that SLIT2 overexpression inhibited the proliferation, migration and invasion of thyroid cancer cells. In addition, a previous study reported that disrupting SLIT2-ROBO signaling in pancreatic ductal adenocarcinoma cells enhanced cancer metastasis and predisposed to neural invasion (33). Collectively, the aforementioned findings support the hypothesis that SLIT2 may serve a role as an inhibitor of cell migration.
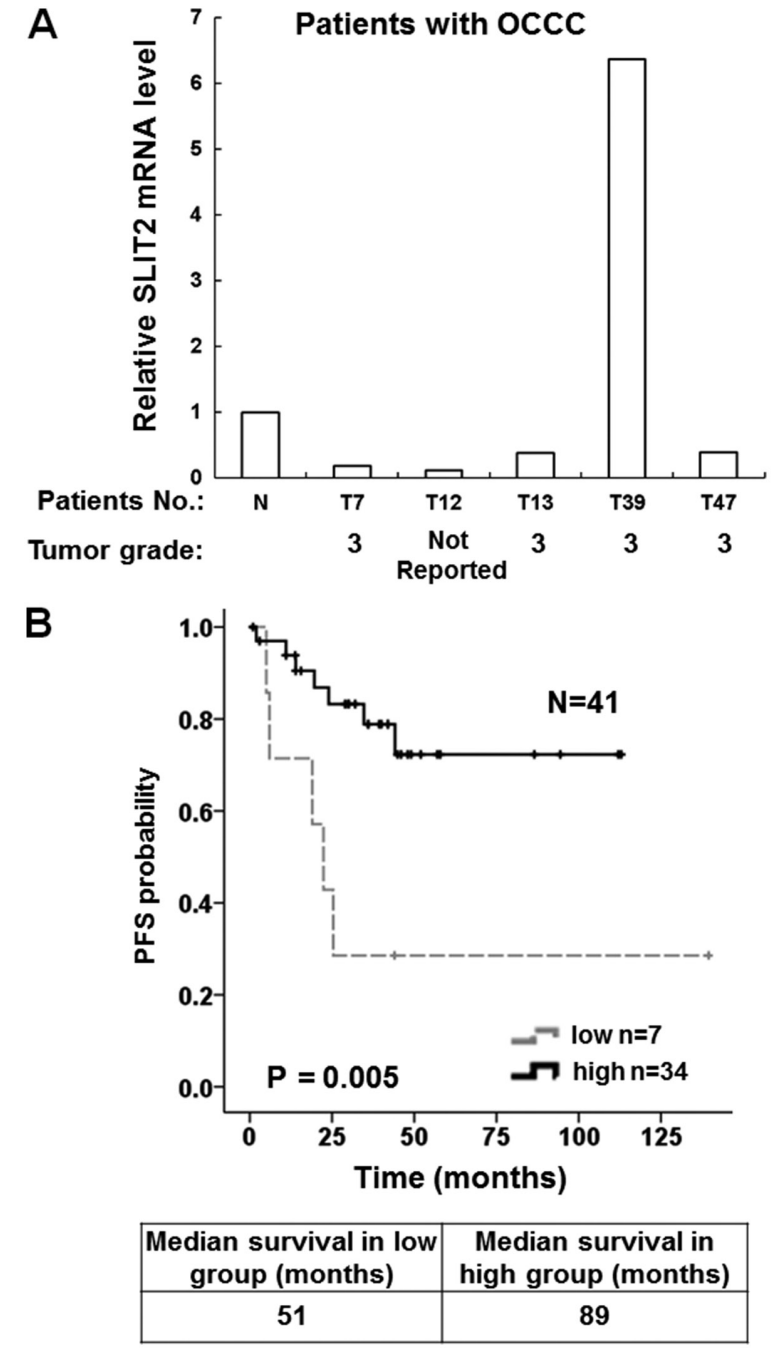

Figure 5. Survival analysis among patients with OCCC associated with SLIT2 mRNA expression. (A) Lower expression levels of SLIT2 were observed in $4 / 5$ of the cDNA T samples compared with the $\mathrm{N}$ samples from tissue array panels (cat. nos. HORT102 and HORT104; OriGene Technologies, Inc.). (B) Low SLIT2 expression was associated with poor PFS in patients with OCCC based on the data of the GSE8841 and GSE65986 projects. SLIT2, slit guidance ligand 2; OCCC, ovarian clear cell carcinoma; N, normal; T, tumor; PFS, progression-free survival.

In the present study, the expression levels of SLIT2 in OCCC were found to be associated with the expression levels of $\beta$-catenin and pAKT, which is in line with the results of studies targeting breast and lung cancer $(19,34)$. Additionally, the current findings demonstrated that the protein expression levels of $\beta$-catenin, pAKT and SNAI1 were increased in OCCC cells treated with shRNA-SLIT2. Conversely, low protein expression levels of $\beta$-catenin, pAKT and SNAI1 were identified in OCCC cells when SLIT2 was overexpressed. The EMT in ovarian cancer is induced by multiple factors, including hepatocyte growth factor (HGF) signaling (35). A previous study demonstrated that shRNA-SLIT2 was able to enhance HGF-induced migration, while overexpression of SLIT2 was able to inhibit HGF-triggered motile responses in SKOV3 cells (36). Thus, it is worth investigating whether there are other signaling pathways that are associated with SLIT2 signaling in order to further understand the role of SLIT2. SLIT2 is a secreted 
glycoprotein and its expression appears to be frequently downregulated in various types of human cancer, such as esophageal, thyroid and lung cancer $(10,31,32,34)$. Thus, the SLIT2 peptides may represent a new target when designing novel therapeutic molecules that may help to attenuate human OCCC metastasis.

\section{Acknowledgements}

The authors would like to thank Dr Tang-Yuan Chus (Department of Obstetrics and Gynecology, Tzu Chi General Hospital, Hualien, Taiwan) for his helpful discussions regarding this research.

\section{Funding}

The present study was supported in part by the Ministry of Science and Technology (grant no. MOST NSC101-2320-B-320-008) and the Buddhist Tzu Chi Medical Foundation (grant no. TCMMP108-01-03).

\section{Availability of data and materials}

The datasets generated and/or analyzed during the current study are available in the Gene Expression Omnibus repository (https://www.ncbi.nlm.nih.gov/geo/query/acc.cgi).

\section{Authors' contributions}

CJL performed the experiments and participated in the analysis of the data. WRH participated in the acquisition and analysis of the data. CZW performed the clinical experiments. RCT designed this study, analyzed the data and drafted the manuscript. CJL and RCT confirm the authenticity of all the raw data. All authors read and approved the final manuscript.

\section{Ethics approval and consent to participate}

Not applicable.

\section{Patient consent for publication}

Not applicable.

\section{Competing interests}

The authors declare that they have no competing interests.

\section{References}

1. Siegel RL, Miller KD and Jemal A: Cancer statistics, 2020. CA Cancer J Clin 70: 7-30, 2020.

2. Website of Health Promotion Administration, Ministry of Health and Welfare, Taiwan. 2019 Available online: https://www.hpa.gov.tw/Pages/ashx/File.ashx?FilePath= /File/ Attach/10227/File_11644.pdf

3. Tan DS, Miller RE and Kaye SB: New perspectives on molecular targeted therapy in ovarian clear cell carcinoma. Br J Cancer 108 $1553-1559,2013$

4. Shaw TJ, Senterman MK, Dawson K, Crane CA and Vanderhyden BC: Characterization of intraperitoneal, orthotopic, and metastatic xenograft models of human ovarian cancer. Mol Ther 10: 1032-1042, 2004.
5. Torre LA, Trabert B, DeSantis CE, Miller KD, Samimi G, Runowicz CD, Gaudet MM, Jemal A and Siegel RL: Ovarian cancer statistics, 2018. CA Cancer J Clin 68: 284-296, 2018.

6. Seyfried TN and Huysentruyt LC: On the origin of cancer metastasis. Crit Rev Oncog 18: 43-73, 2013.

7. Okamoto A: Management and new strategy of ovarian clear cell carcinoma. Int J Clin Oncol 25: 418, 2020.

8. Chaffer CL and Weinberg RA: A perspective on cancer cell metastasis. Science 331: 1559-1564, 2011.

9. Jiang L, Wang Y, Rong Y, Xu L, Chu Y, Zhang Y and Yao Y: MiR-1179 promotes cell invasion through SLIT2/ROBO1 axis in esophageal squamous cell carcinoma. Int J Clin Exp Pathol 8: 319-327, 2015.

10. Tseng RC, Chang JM, Chen JH, Huang WR, Tang YA, Kuo IY, Yan JJ, Lai WW and Wang YC: Deregulation of SLIT2-mediated $\mathrm{Cdc} 42$ activity is associated with esophageal cancer metastasis and poor prognosis. J Thorac Oncol 10: 189-198, 2015.

11. Jiang Z, Liang G, Xiao Y, Qin T, Chen X, Wu E, Ma Q and Wang Z: Targeting the SLIT/ROBO pathway in tumor progression: Molecular mechanisms and therapeutic perspectives. Ther Adv Med Oncol 11: 1758835919855238, 2019.

12. Lucas B and Hardin J: Mind the (sr)GAP-roles of Slit-Robo GAPs in neurons, brains and beyond. J Cell Sci 130: 3965-3974, 2017.

13. Blockus H and Chédotal A: Slit-Robo signaling. Development 143 3037-3044, 2016.

14. Qiu H, Zhu J, Yu J, Pu H and Dong R: SLIT2 is epigenetically silenced in ovarian cancers and suppresses growth when activated. Asian Pac J Cancer Prev 12: 791-795, 2011.

15. Dai CF, Jiang YZ, Li Y, Wang K, Liu PS, Patankar MS and Zheng J: Expression and roles of Slit/Robo in human ovarian cancer. Histochem Cell Biol 135: 475-485, 2011.

16. Dong R, Yu J, Pu H, Zhang Z and Xu X: Frequent SLIT2 promoter methylation in the serum of patients with ovarian cancer. J Int Med Res 40: 681-686, 2012.

17. Dickinson RE and Duncan WC: The SLIT-ROBO pathway: A regulator of cell function with implications for the reproductive system. Reproduction 139: 697-704, 2010.

18. Sirohi VK, Popli P, Sankhwar P, Kaushal JB, Gupta K, Manohar M and Dwivedi A: Curcumin exhibits anti-tumor effect and attenuates cellular migration via Slit-2 mediated down-regulation of SDF-1 and CXCR4 in endometrial adenocarcinoma cells. J Nutr Biochem 44: 60-70, 2017.

19. Chang PH, Hwang-Verslues WW, Chang YC, Chen CC, Hsiao M, Jeng YM, Chang KJ, Lee EY, Shew JY and Lee WH: Activation of Robol signaling of breast cancer cells by Slit2 from stromal fibroblast restrains tumorigenesis via blocking PI3K/Akt/ $\beta$-catenin pathway. J Cancer Res 72: 4652-4661, 2012.

20. Yeh YM, Chuang CM, Chao KC and Wang LH: MicroRNA-138 suppresses ovarian cancer cell invasion and metastasis by targeting SOX4 and HIF-1 $\alpha$. Int J Cancer 133: 867-878, 2013

21. Livak KJ and Schmittgen TD: Analysis of relative gene expression data using real-time quantitative PCR and the 2(-Delta Delta C(T)) method. Methods 25: 402-408, 2001.

22. Marchini S, Mariani P, Chiorino G, Marrazzo E, Bonomi R, Fruscio R, Clivio L, Garbi A, Torri V, Cinquini M, et al: Analysis of gene expression in early-stage ovarian cancer. Clin Cancer Res 14: 7850-7860, 2008

23. Makii C, Oda K, Sone K, Sone K, Hasegawa K, Uehara Y, Nishijima A, Asada K, Koso T, Fukuda T, et al: MDM2 is a potential therapeutic target and prognostic factor for ovarian clear cell carcinomas with wild type TP53. Oncotarget 7: 75328-75338, 2016.

24. Yamaguchi K, Huang Z, Matsumura N, Mandai M, Okamoto T, Baba T, Konishi I, Berchuck A and Murphy SK: Epigenetic determinants of ovarian clear cell carcinoma biology. Int J Cancer 135: 585-597, 2014.

25. Kang J, Shin SH, Yoon H, Huh J, Shin HW, Chun YS and Park JW: FIH is an oxygen sensor in ovarian cancer for G9a/GLP-Driven epigenetic regulation of metastasis-related genes. Cancer Res 78: 1184-1199, 2018.

26. Chang JM, Tsai AC, Huang WR and Tseng RC: The alteration of CTNNBIP1 in lung cancer. Int J Mol Sci 20: 5684, 2019.

27. Lamouille S, Xu J and Derynck R: Molecular mechanisms of epithelial-mesenchymal transition. Nat Rev Mol Cell Biol 15: 178-196, 2014.

28. Puisieux A, Brabletz T and Caramel J: Oncogenic roles of EMT-inducing transcription factors. Nat Cell Biol 16: 488-494, 2014. 
29. Wang LH, Wu CF, Rajasekaran N and Shin YK: Loss of tumor suppressor gene function in human cancer: An overview. Cell Physiol Biochem 51: 2647-2693, 2018.

30. Dickinson RE, Myers M and Duncan WC: Novel regulated expression of the SLIT/ROBO pathway in the ovary: Possible role during luteolysis in women. Endocrinology 149: 5024-5034, 2008.

31. Dickinson RE, Fegan KS, Ren X, Hillier SG and Duncan WC: Glucocorticoid regulation of SLIT/ROBO tumour suppressor genes in the ovarian surface epithelium and ovarian cancer cells. PLoS One 6: e27792, 2011.

32. Jeon MJ, Lim S, You MH, Park Y, Song DE, Sim S, Kim TY, Shong YK, Kim WB and Kim WG: The role of Slit2 as a tumor suppressor in thyroid cancer. Mol Cell Endocrinol 483: 87-96, 2019.

33. Göhrig A, Detjen KM, Hilfenhaus G, Körner JL, Welzel M, Arsenic R, Schmuck R, Bahra M, Wu JY, Wiedenmann B and Fischer C: Axon guidance factor SLIT2 inhibits neural invasion and metastasis in pancreatic cancer. Cancer Res 74: 1529-1540, 2014
34. Tseng RC, Lee SH, Hsu HS, Chen BH, Tsai WC, Tzao C and Wang YC: SLIT2 attenuation during lung cancer progression deregulates beta-catenin and E-cadherin and associates with poor prognosis. Cancer Res 70: 543-551, 2010.

35. Vergara D, Merlot B, Lucot JP, Collinet P, Vinatier D, Fournier I and Salzet M: Epithelial-mesenchymal transition in ovarian cancer. Cancer Lett 291: 59-66, 2010.

36. Stella MC, Trusolino L and Comoglio PM: The Slit/Robo system suppresses hepatocyte growth factor-dependent invasion and morphogenesis. Mol Biol Cell 20: 642-657, 2009.

cc) (i) $\odot$ This work is licensed under a Creative Commons Attribution-NonCommercial-NoDerivatives 4.0 International (CC BY-NC-ND 4.0) License. 